Ann. Zootech., I980, 29 (I), 39-54.

\title{
Productivité numérique annuelle des truies entre 1972 et 1978 estimée à partir des résultats de reproduction et de sevrage
}

\author{
A. AUMAITRE et J. DAGORN
}

Station de Recherches sur l'Elevage des Porcs, Centre national de Recherches zootechniques, I.N.R.A., $7835^{\circ}$ Jouy-en-Josas, France

Institut Technique du Porc, 149, me de Bercy

75595 Paris Cedex I2

\section{Résumé}

L'enregistrement des caractéristiques des truies (date de naissance, de mise bas, de sevrage et de réforme) ainsi que celui de leurs performances successives (effectifs des porcelets nés et sevrés) ont été entrepris en France sur les troupeaux porcins à partir de I969. L'évolution. des valeurs moyennes de l'âge au sevrage, de l'intervalle entre le sevrage et la fécondation, de la taille de la portée et de la mortalité post-natale a été suivie et on a calculé sur l'ordinateur de l'I.N.R.A. le nombre de porcelets sevrés par truie productive et par an. Einn I978, les résultats concernent 512832 portées et I I départements sur 82 en contrôlent plus de 10 ooo, soit 64,4 p. I oo du total.

La durée moyenne de lactation a été réduite de I I jours $(41,8$ à 30,9$)$ en 6 ans, l'écart sevrage-saillie fécondante diminué de 3,2 jours $(20,2$ à 17). La taille de la portée à la naissance est restée constante alors que le taux moyen de mortalité a été réduit. La productivité numérique moyenne par truie productive et par an a été améliorée de 2, I porcelets entre I972 et I978; 77 p. I oo de ce progrès s'expliquent par la réduction de la durée d'allaitement; les autres paramètres (intervalle sevrage-saillie fécondante et mortalité) expliquent seulement o,5 porcelet par truie et par an. Des progrès sont enregistrés dans tous les départements : ainsi l'écart maximum entre les moyennes départementales qui était de $3, \mathrm{I}$ porcelets (I4,9 à I8) en I972 a été réduit à 2,5 animaux par truie productive et par an $\left(18,5\right.$ à 20,5) en $197^{8}$.

I,es possibilités d'amélioration nouvelles sont liées à une meilleure maitrise au niveau des élevages, de techniques déjà éprouvées dans des conditions expérimentales. La possibilité d'une rapide diffusion de ces techniques est discutée.

\section{Introduction}

La mesure de la taille de la portée des truies à la naissance et au sevrage a depuis longtemps été retenue pour exprimer la valeur des reproducteurs dans l'espèce porcine. Cependant, seuls les livres généalogiques (MolFNAT, I960) enregistraient les performances des truies inscrites afin d'effectuer une sélection sur 
les performances d'élevage (LEGAULT, FELGINEs et OWEN, 1977). Les moyennes calculées sur les seuls animaux inscrits ont une signification et une portée technique limitées car elles concernaient rarement toutes les truies d'un troupeau. Ia nécessité économique de produire un plus grand nombre de portées par an grâce à la réduction de la durée des cycles de reproduction a été récemment rappeléc (ENCLISH, SMith et MAC LEAN, I977).

La durée de lactation atteignait en I970 couramment deux mois dans de nombreux élevages, conformément aux recommandations séculaires de l'Abbé ROZIER (I785), et 1'on attachait peu d'importance à une saillie à l'ostrus qui suivait immédiatement le sevrage. Ces dernières années, l'élevage porcin a bear1coup évolué, En effet au plan économique, 6 ooo $\mathrm{F}$ d'investissements sont nécessaires pour le logement d'une truie et de ses porcelets, ainsi que 2 tonnes d'aliment concentré et 24 heures de main d'œuvre dévouée et qualifiée par an (BUYLE 1976; Pa.queT et $a l$., I976). La rentabilité de l'élevage (ENGEL KE, I969; DACORN, FERRADINI et DE JUBECOURT, I978) ne peut être assurée que par la production d'un nombre croissant de porcelets par truie et par an ou productivité (ORTAVANT et Thibault, I97I). Or de nombreux travaux expérimentaux ont été réalisés sur l'allaitement et le sevrage des porcelets à 5 semaines (AUMAITRE et SAI MONLEGAGNEUR, I96I; SALMON-LEGAGNEUR et JOUANDET, I962), puis sur le sevrage à 3 semaines (SElF et Grummer, I958; Aumaitre et RetTtagliatu, I972; Maury, PALISSE et POLINE, I973; TE BRAKE, I978). Tous permettaient d'envisager la réuscite du sevrage précoce au niveau des élevages, entrainant ainsi une réduction de la durée de lactation et une augmentation sensible de la fréquence des misebas. Des progrès dans 1a maîtrise de l'œstrus et de la reproduction de la truie ont été également réalisés (DU MESNIL DU Buisson, MAUlíon et JoNDET, I97I) permettant de réduire l'intervalle entre le sevrage et une nouvelle fécondation.

Il restait à vérifier que ces recommandations étaient appliquées avec succès a11 niveau des élevages. Un programme de gestion technique des élevages de truies a été réalisé en I.969 dans le but de mesurer les performances moyennes de chaque troupeau et de suivre l'évolution de la productivité numérique par truie. On dispose ainsi de références techniques successives par groupements de producteurs et par département, et on peut proposer une amélioration des résultats par une meilleure maîtrise des composantes de la productivité (I)AGoRN, I975).

\section{Matériel et méthodes}

L'enregistrement des performances successives des truies identifiées à l'intérieur de chaque troupeau d'effectif supérieur à 20 truies a été entrepris à l'initiative de 1'I.N.R.A. et de 1'I.T.P. avec le concours de 1'Association Nationale pour le Développement Agricole (ANDA) et du Fonds d'Orientation et de Régularisation des Marchés Agricoles (FORMA). Les organismes départementaux (FDE, SUAD, groupements de producteurs) assurent la collecte et le contrôle des données de base. Les dates de naissance, de mise bas et de sevrage ainsi que la taille de la portée à la naissance et au sevrage sont recueillies tous les trimestres dans les élevages (DAGORN, I975). L es valeurs moyennes calculées concernent l'âge au sevrage, l'intervalle sevrage-fécondation (pour les truies mettant bas au moins deux portées). La productivité numérique est exprimée par les deux formules suivantes : 
SEVRAGE ET PRODUCTIVITÉ DES TRUIES

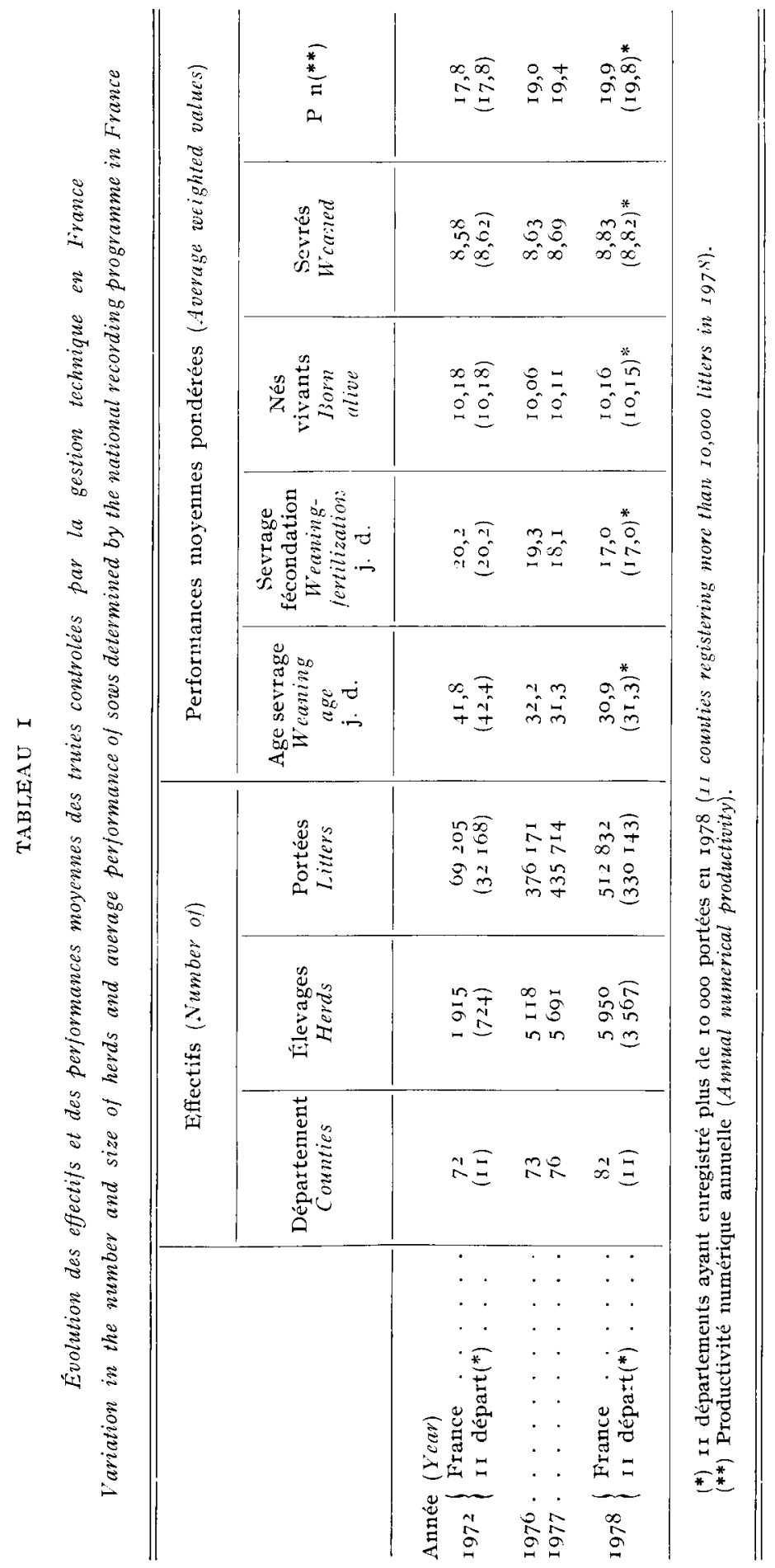


- soit par truie productive $(\mathrm{P} n)$ :

$$
\mathrm{P} n=\frac{\mathrm{T} p(\mathrm{I}-\mathrm{T} m) 365}{\mathrm{G}+\mathrm{L}+\mathrm{I}_{s f}},
$$

Legault, Aumaitre et du Mesnil du Butsson, I975.

- soit par truie présente dans le troupeau entre 1'âge de 200 jours et la réforme $\left(\mathrm{P}^{\prime} n\right)$ :

$$
\mathrm{P}^{\prime} n=\frac{\mathrm{N} \mathrm{T} p(\mathrm{I}-\mathrm{T} m) 365}{i_{1}+\mathrm{I}(\mathrm{N}-\mathrm{I})+i_{2}}, \text { LEGAULT, I } 978 .
$$

où T $p$ est le nombre moyen de porcelets nés vivants par portée, T $m$ est le taux de mortalité entre la naissance et le sevrage ( $\mathrm{T} m$ varie de o à I pour une inortalité variant de o à I $00 \mathrm{p}$. Ioo);

$\mathrm{G}, \mathrm{L}_{\mathrm{f}}$ et $\mathrm{I}_{s f}$ sont respectivement les durées de gestation, de lactation et l'intervalle entre le sevrage et une nouvelle fécondation;

I est l'intervalle moyen entre mises bas $i_{1}$ l'intervalle entre 200 jours d'âge et la première mise bas et $i_{2}$ l'intervalle entre la dernière mise bas et la réforme.

Les résultats recueillis à l'échelon régional sont centralisés et interprétés sur l'ordinateur de l'I.N.R.A. (C.T.I., Jouy-en-Josas), ils sont régulièrement retournés aux éleveurs et constituent une banque de données disponibles au niveau national. Les moyennes départementales annuelles ont été retenues dans notre étude en choisissant 1972 comme année de référence, ainsi que les mêmes valeurs calculées en I976, I977 et I978. Malgré leur caractère général et la dis-

\section{TABLEAU 2}

Évolution de l'effectif des élevages et du nombre de portées enregistrées dans II départements entre 1972 et 1978 . Variation in the number of herds and registered litters in 1 I connties between 1972 and 1978 .

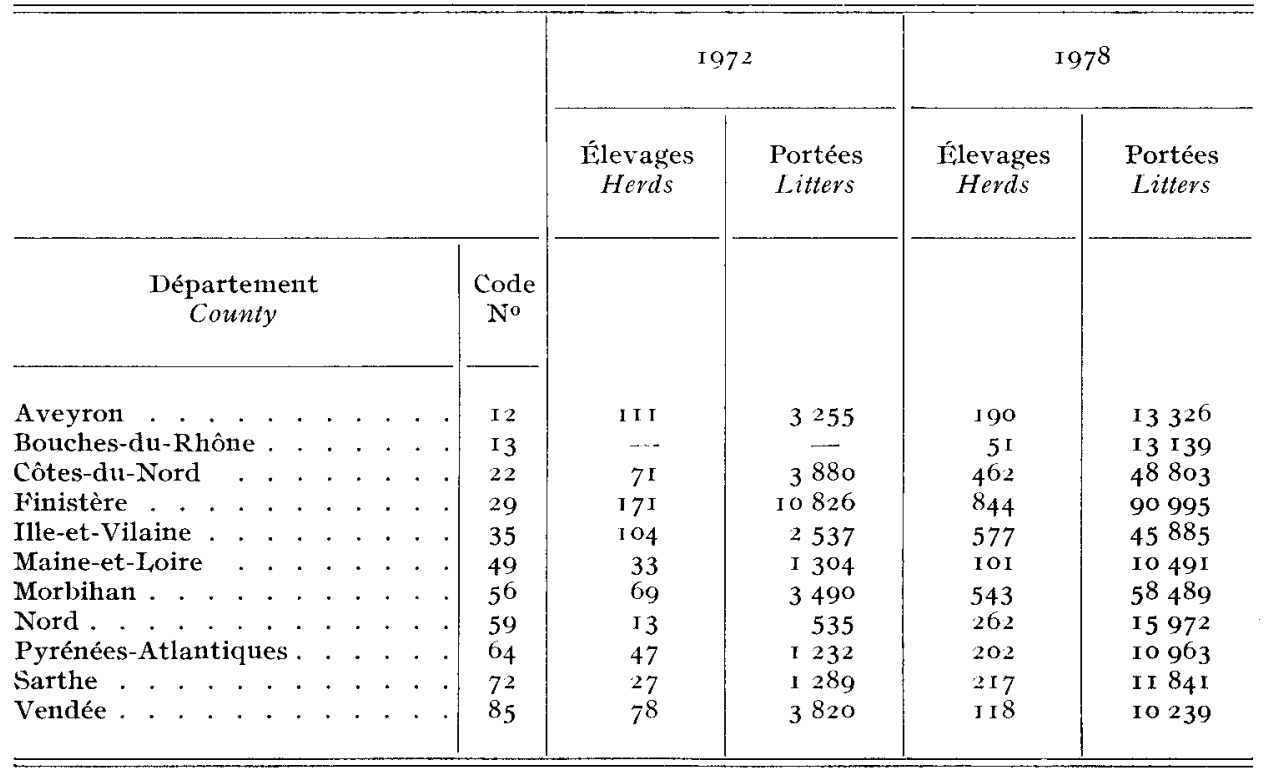


persion possible intradépartement (entre élevages) nous avons cherché à interpréter l'évolution de chaque composante de la productivité sur plusieurs années : durée de lactation, intervalle sevrage-saillie fécondante, effectifs à la naissance et au sevrage et taux de mortalité.

Le nombre des départements concernés évolue peu, mais le nombre d'élevages et de portées enregistrées (tabl. I) augmente surtout entre I972 et r976. En 6 ans (1972-1978), le nombre moyen de portées sevrées par élevage et par an est passé de 36 à 86 . Toutefois, une variabilité très grande existe encore en I 978 entre départements : le département du Finistère a contrôlé 90995 portées, celui du Rhône I29 seulement. Aussi, nous avons analysé en détail l'évolution des performances moyennes des I I départements ayant collecté plus de ro ooo portées en I 978 (tabl. 2). Sur ces bases, nous nous proposons de discuter de l'évolution des performances noyennes des truies, des progrès accomplis et de ce qu'il est encore possible de réaliser au niveau des élevages.

\section{Résultats}

\section{I. - Utilisation de la gestion technique par les éleveurs}

A partir de 1969, quelques milliers de portées issues des meilleurs élevages ont été enregistrées. L'échantillon national était peu stable, en raison de l'augmentation de la taille et du nombre des élevages contrôlés et de l'extension des

TABLEAU 3

Evolution des paramètres de la productivité moyenne observée entre 1972 and 1978 Variation in the parameters of sow productivity between 1972 and 1978

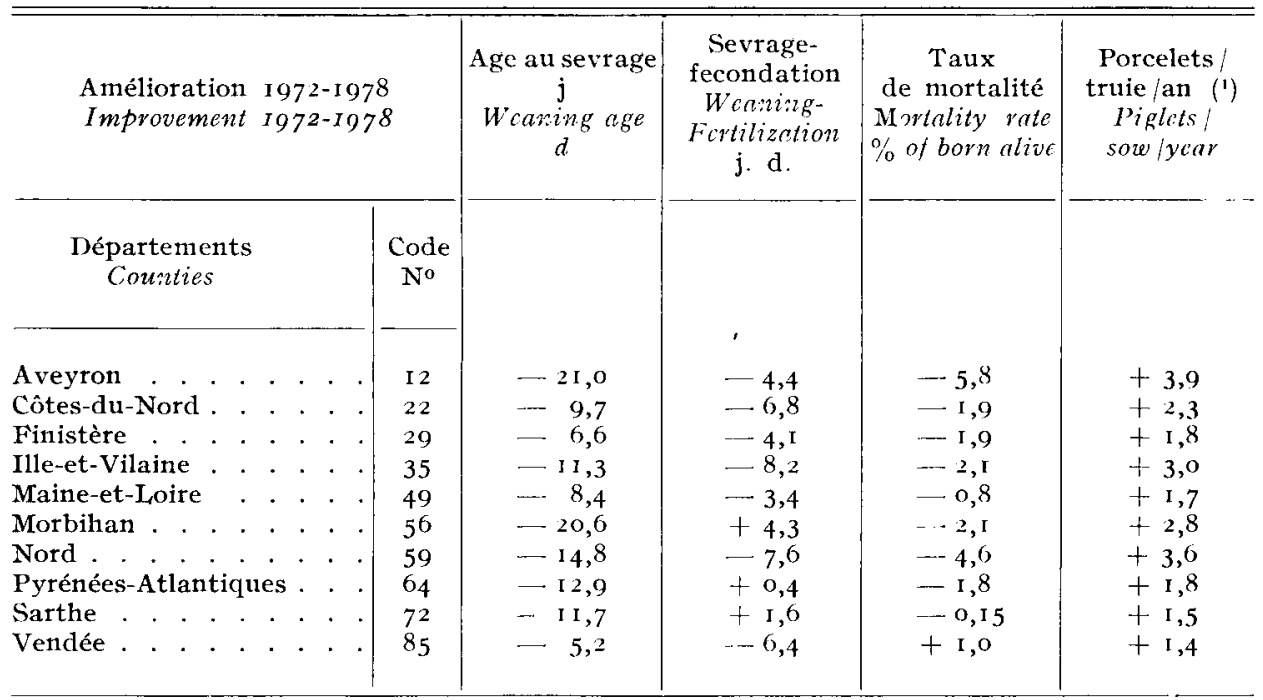

(1) Gain de productivité : porcelets sevrés/truie productive/an (Productivily gain: weaned piglets/ productive sow /year). 
enregistrements à l'ensemble des départements. Dès I972, 69000 portées ont été enregistrées et en I978 près de 5I3 ooo enregistrements sont disponibles (tabl. I). On observe donc une multiplication par 7,4 des effectifs suivis en 6 ans, alors que celui des élevages contrôlés a été multiplié par 3 seulement. Les relevés sont effectués sur tout le territoire français ( 82 départements). La "production " des 4 départements de la région de programme Bretagne fournit 48 p. roo des données (tabl. 2) et celle des II principaux départements 64,4 p. Ioo des déclarations pour 3567 élevages en I 978 , Douze autres départements déclarent entre 5 or6 (Seine-Maritime) et 8783 (Loire-Atlantique) portées par an, soit 75732 portées supplémentaires élevées dans 897 élevages.

Peu d'élevages abandonnent les enregistrements au cours de la période, ce qui donne un caractère de continuité et d'homogénéité à l'échantillon et une signification statistique aux résultats observés pendant plusieurs années.

\section{II. - Évolution de l'âge moyen au sevrage}

L'examen de l'ensemble des moyennes des départements (fig. 1) révèle en I972 que dans plus de la moitié des observations ( 45 sur 72 ) le sevrage des porcelets

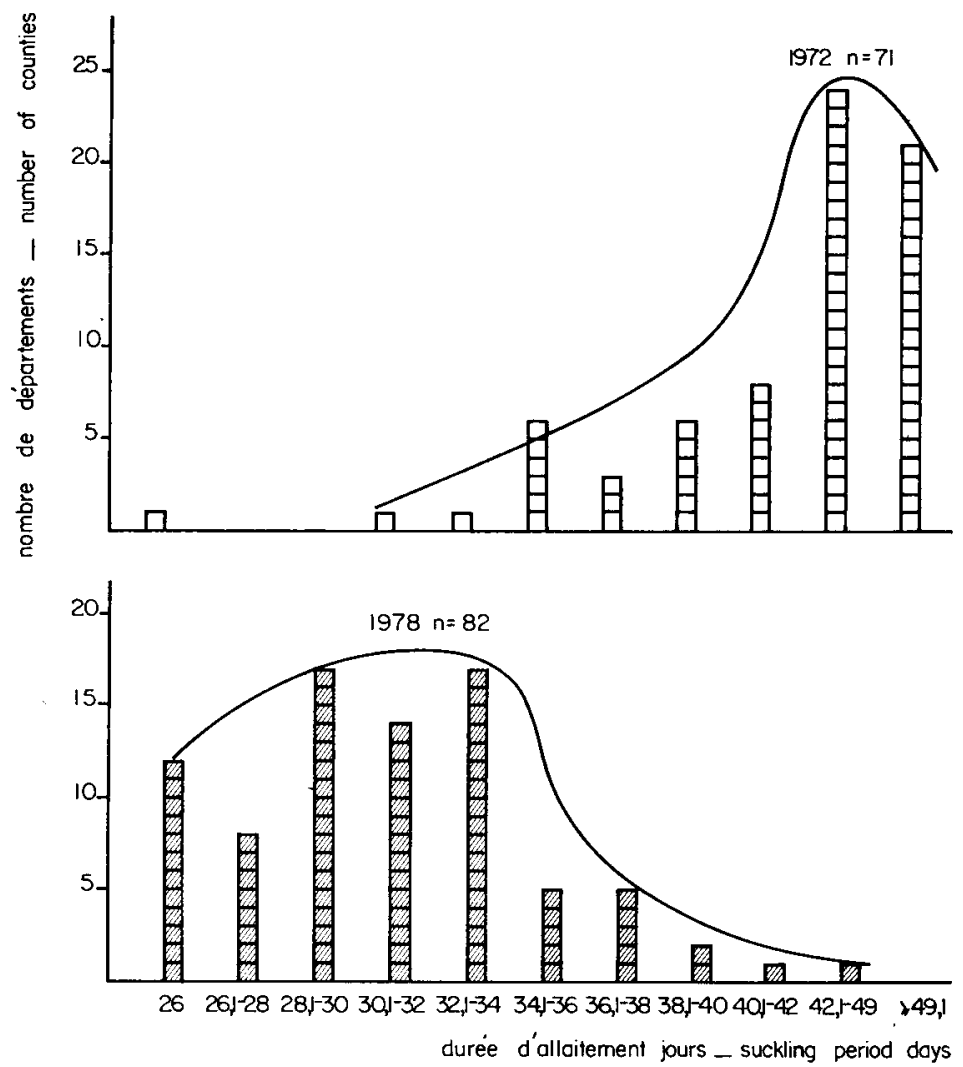

Fig. I. - Répartition des moyennes départementales de la durée d'allaitement des porcelets entre 1972 et 1978 .

Length of the suckling period for piglets between $197^{2}$ and 1978 . County mean frequency. 
est pratiqué au-delà de 42 jours et même pour $2 \mathrm{I}$ d'entre eux après 49 jours. En 1978 au contraire, dans une minorité soit $\mathrm{I}_{4}$ sur 82 , on sèvre au-delà de 34 jours. A l'opposé, la moyenne est inférieure à 26 jours dans $\mathrm{I} 2$ départements. Enfin 37 départements représentant I62 975 portées ( $32 \mathrm{p}$. Ioo de l'effectif déclaré) ont un âge au sevrage inférieur à 30 jours en 1978 .

Les moyennes nationales pondérées pour l'effectif départemental nous montrent (tabl. I) que la réduction de la durée de l'allaitement a représenté ro,9 jours en 6 ans; l'évolution a été surtout sensible entre 1972 et 1976 ( $-9,6$ jours); depuis, l'âge moyen au sevrage tend vers un mois. Sur l'échantillon de référence, on constate une réduction du même ordre, variable d'un département à l'autre. Ainsi (tab1. 3, fig. 2) les éleveurs de 1'Aveyron, du Morbihan et du Nord ont adopté plus rapidement le sevrage précoce, et dès I 976 toutes les moyennes correspondantes sont en dessous de 35 iours. Toutefois, la durée de lactation évolue peu au cours des trois dernières années sauf en Aveyron et en Ille-et-Vilaine où elle est sensiblement réduite. Ainsi, le sevrage est maintenu à 35 jours dans le Nord et à 33 jours dans le Finistère alors qu'il s'effectue vers l'âge de I mois pour plus de r ro ooo portées de trois autres départements (Aveyron, Côtes-du-Nord, Ille-et-Vilaine).

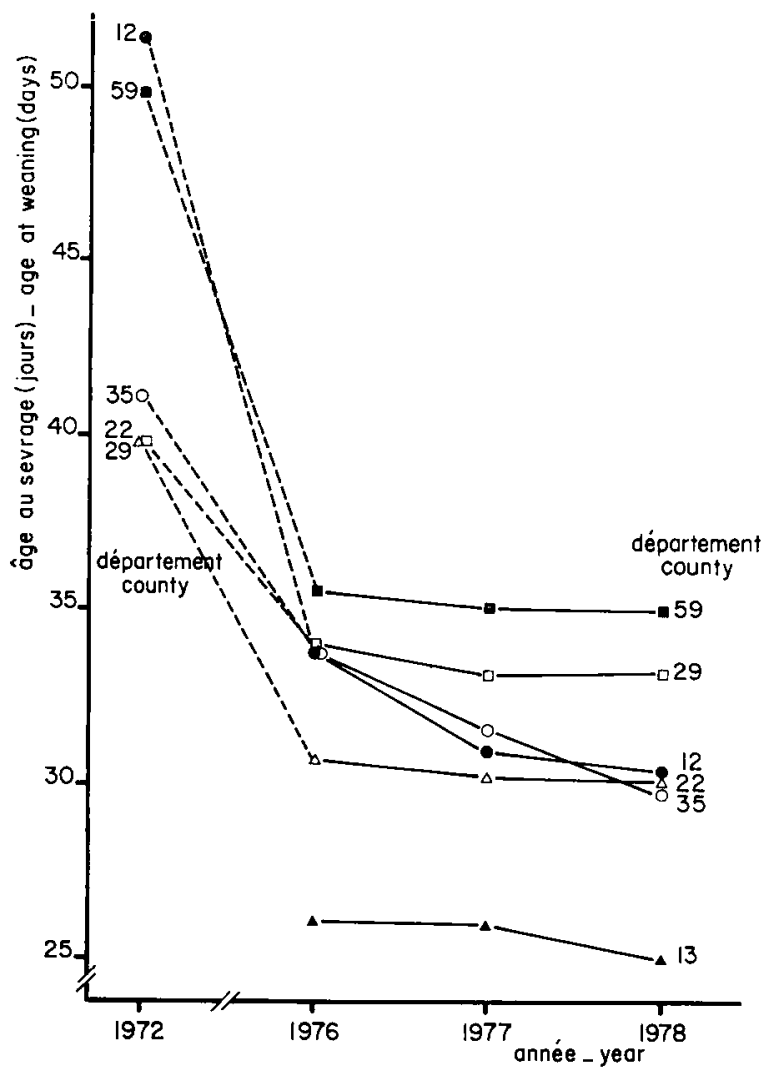

FIG. 2. - Age moyen au sevrage des porcelets depuis 1972 et au cours des trois dcriières années. Average weaning age of piglets in $197^{2}$ and during the last 3 years 


\section{II1. - Intervalle sevrage saillie fécondante}

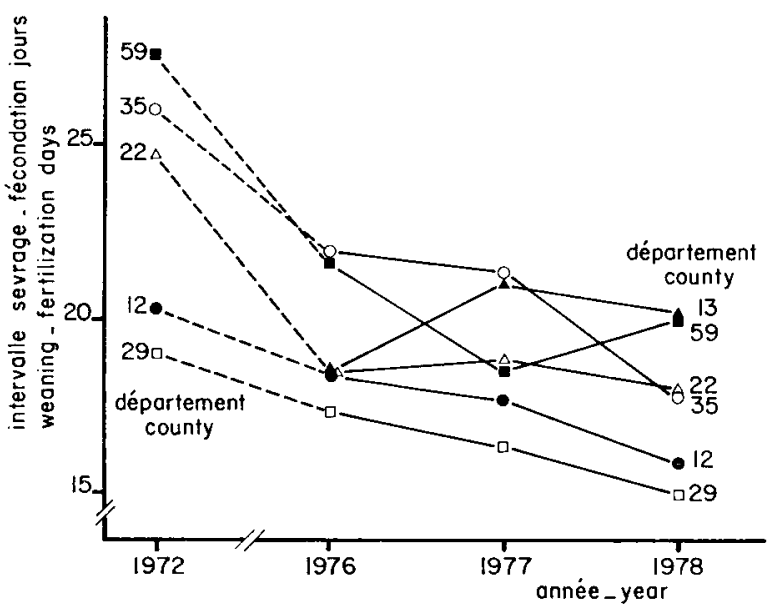

FIG. 3. - Evolution de l'intervalle moyen Sevrage-Fécondation depuis $197^{2}$ et au cours des 3 dernières années.

Average weaning fertilization interval in 1972 and during the last 3 years

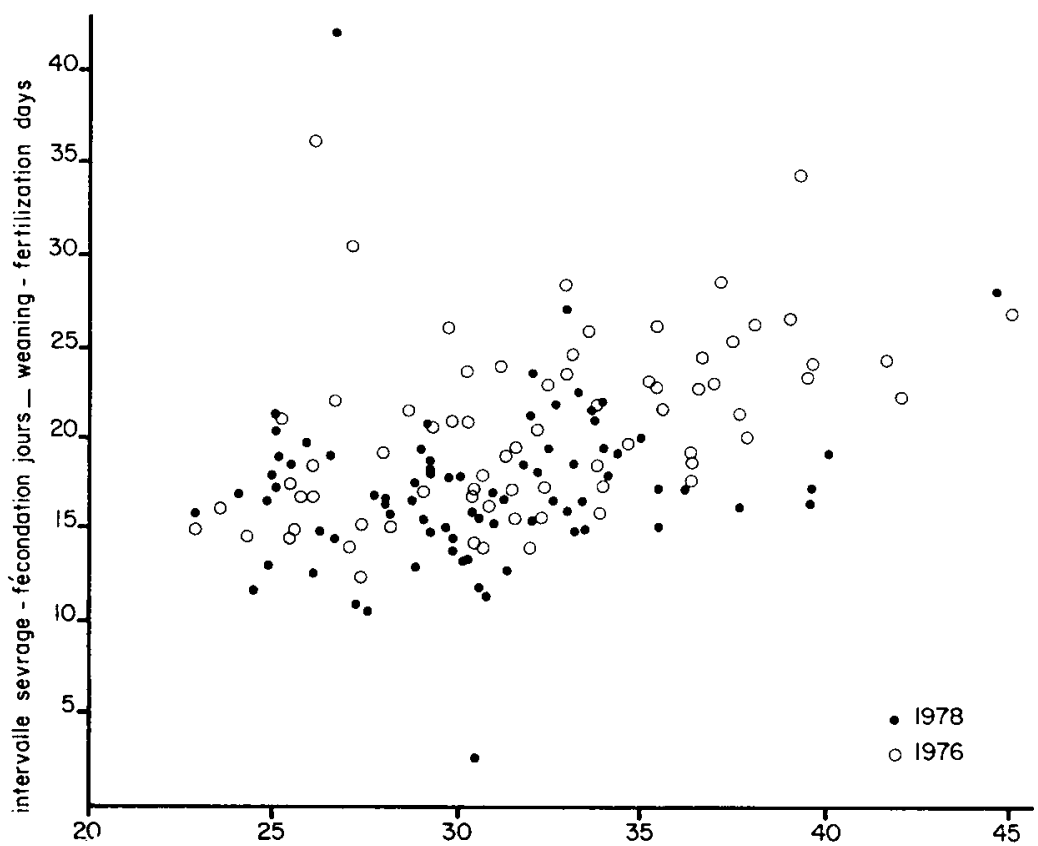

I'IG. 4. - Relations entre la durée moyenne d'allaitement $(x)$ et l'intervalle Sevrage-Fécondation (y) (2 moyennes anormales sont supprimées).

Relationship between average lactation length $(x)$ and weaning-fertile mating interval $(y) .2$ erratic values weve discarded.

$$
\begin{aligned}
& 1976 r=+0,563 \quad \text { P < 0,01; } y=0,8313 \quad x-5,7 \\
& 1978 r=+0,254 \quad \mathrm{P}<0,05 ; \quad y=0,3309 x+7,49
\end{aligned}
$$


I restent anormalement élevées ( $\mathrm{I}_{7}$ jours en $\mathrm{I}_{97} 8$ ). Filles recouvrent 1'intervalle sevrage-cestrus, l'incidence des retours en chaleur et des avortements précoces, et enfin parfois, les truies ayant perdu tous leurs porcelets à la naissance. Toutefois, on observe une tendance à une réduction constante de cet intervalle : entre 1972 et 1978 , le nombre des départements où la durée moyenne de l'intervalle dépassait 20 jours est passée de 39 à I 5 . Au niveau du troupeatı national, une diminution de 3,2 jours de l'intervalle sevrage fécondation est observée en 6 ans. Dans les I I départements de rétérence, la miême tendance apparaît (tab1. I), mais en I 972 (fig. 3) les valeurs extrêmes étaient comprises entre ro jours (Finistère) et 27,6 (Nord). Pour les Côtes-du-Nord et le Nord, la diminution initiale rapide jusqu'en r 976 ne s'est pas poursuivie; par contre un effort constant est observé en Ille-etVilaine, et surtout en Aveyron et dans le Finistère. Paradoxalement certaines moyennes augmentent entre 2 observations (Morbihan, Pyrénées Atlantiques, Sarthe) avec la prise en compte de nombreux élevages nouveaux (tabl. 2). L'effort initial semble donc porter sur la réduction de la durée de l'allaitement plutôt que sur le contrôle de la rcproduction des truies.

Nous avons également exprimé la relation entre la durée de lactation et l'écart sevrage-fécondation. L'expression graphique des résultats moyens par département (fig. 4) montre une légère tendance à l'augmentation du déiai de fécondation au fur et à mesure que la durée de lactation augmente. Les cofficients de corrélation calculés entre les deux paramètres montrent une liaison linéaire positive et significative $(r=+0,56$ en 1976 et $r=+0,25$ en 1978), qui mérite d'être soulignte.

\section{IV. -.. Taille de la portée et taux de mortalité}

A partir des enregistrements moyens pondérés, on constate une remarquable stabilité de la taille moyenne de la portée à la naissance, soit $\longrightarrow 0,02$ porcelet

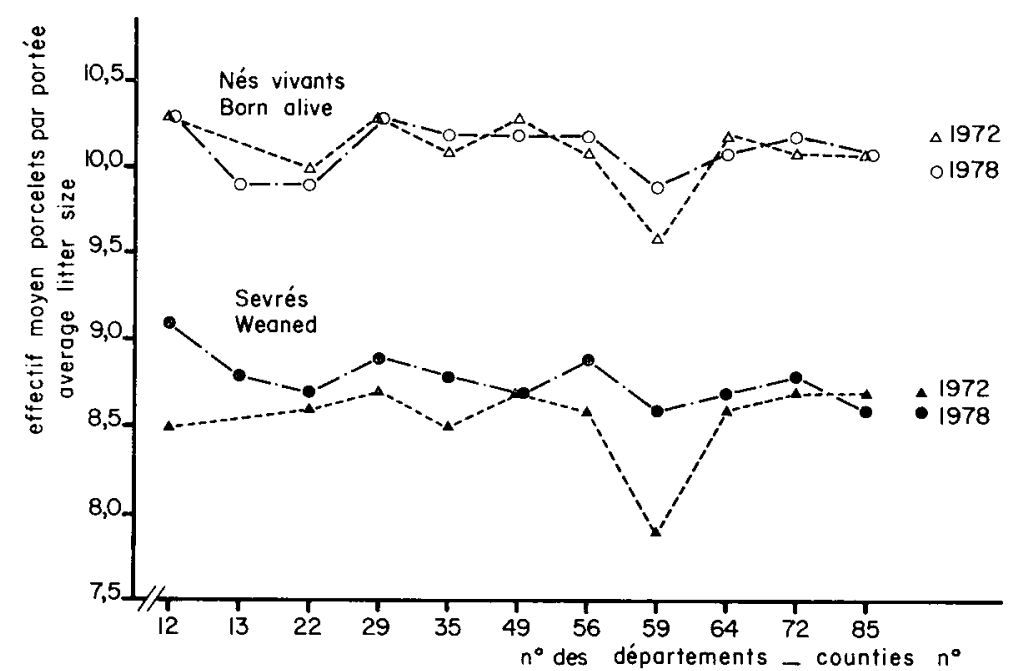

FiG. 5. - Taille moyenne de la portée à la naissance et an sevrage dans i I départements envegistrant ro ooo données.

Average litter size at birth and weaning in II counties between 1972 and 1978 . 
nés vivants entre I972 et I978 (fig. 5). Un accroissement de la taille de la portée à la naissance $(+0,3$ porcelet) et au sevrage $(+0,7$ porceiet $)$ est observé dans le seul département du Nord. Un gain de 0,25 porcelet sevré par portée est également noté (soit un gain supplémentaire théorique annuel de I28 250 animaux sur l'échantillon à la suite d'une baisse du taux moyen de mortalité (tabl. I). Ainsi le taux de survie à 42 jours qui était de 84,3 p. Ioo des animaux nés vivants en 1972 est de 86,9 p. Ioo au sevrage à 30 jours environ en I978. Le taux de mortalité évolue différemment suivant les départements; il a été ramené de 17,5 à II,7 p. Ioo dans le département de 1'Aveyron et a par contre faiblement augmenté en Vendée (I3,9 à I4,9 p. Ioo) dans le mếme temps (tabl. 3).

\section{V. - Évolution de la productivité numérique annuelle}

L'amélioration de la productivité numérique moyenne résulte de l'évolution des 3 paramètres envisagés précédemment. Les performances par truie productive sont passées de I7,8 à I 9,9 porcelets sevrés par truie et par an, soit un gain de

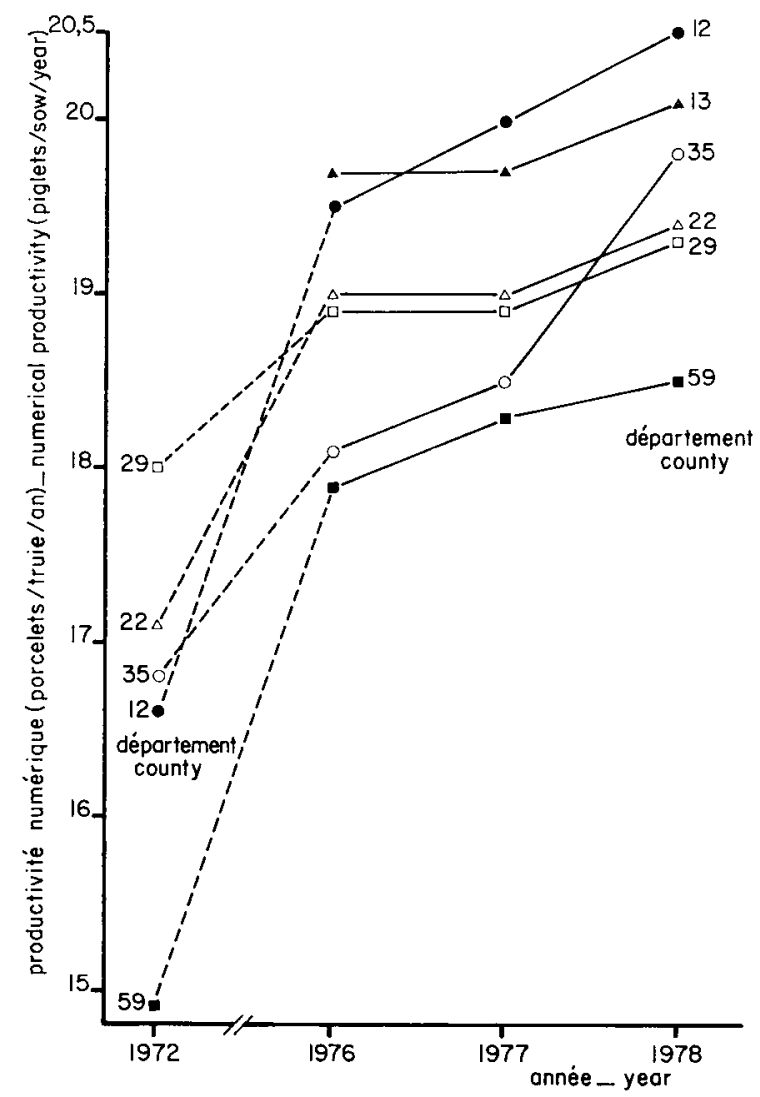

FIG. 6. - Evvolution de la productivité numérique annuelle (Pn) exprimie par le nombre de porcelets sevrés par truie productive.

Average numerical productivity expressed by the number of zeaned piglets per productive sow/year. 
2, I animaux entre $197^{2}$ et $197^{8}$ (tabl. I). De plus, la faible productivité initiale est associée à une variabilité considérable des performances entre départements : I 4,9 à I 8 porcelets en I 972 contre I 8,5 à 20,5 en I 978 (fig. 6). L'essentiel des progrès a été accompli entre r 972 et r976. Depuis, plusieurs tendances apparaissent : la productivité plafonne dans certains départements (Bouches-du-Rloône, Côtesdu-Nord, Finistère) alors qu'elle progresse constamment en A:eyr on, dans le Nord et surtout en Ille-et-Vilaine où plus d'un porcelet a été gargné par truie en un an sur près de 50 ooo portées.

La productivité par truie présente est inférieure à la productivité par truie en production en raison notamment de la prise en compte en I978, d'un délai moyen voisin de 60 jours entre le dernier sevrage et la réforme. L'écart moyen de 2,2 porcelets pour l'Aveyron et les Pyrénées Atlantiques est réduit à I, 8 seulement dans les Bouches-du-Rhône. Toutefois, on observe un certain parallélisme entre les valeurs calculées pour les II départements (fig. 7). La productivité moyenne est égale à $\mathrm{I} 7,54$ porcelets par truie présente et par an pour l'ensemble de l'échantillon et $\mathrm{I} 7,45$ pour les I I départements de référence; elle varie de I6, I (Pyrénćes Atlantiques) à 18,3 porcelets (Bouches-du-Rhône).

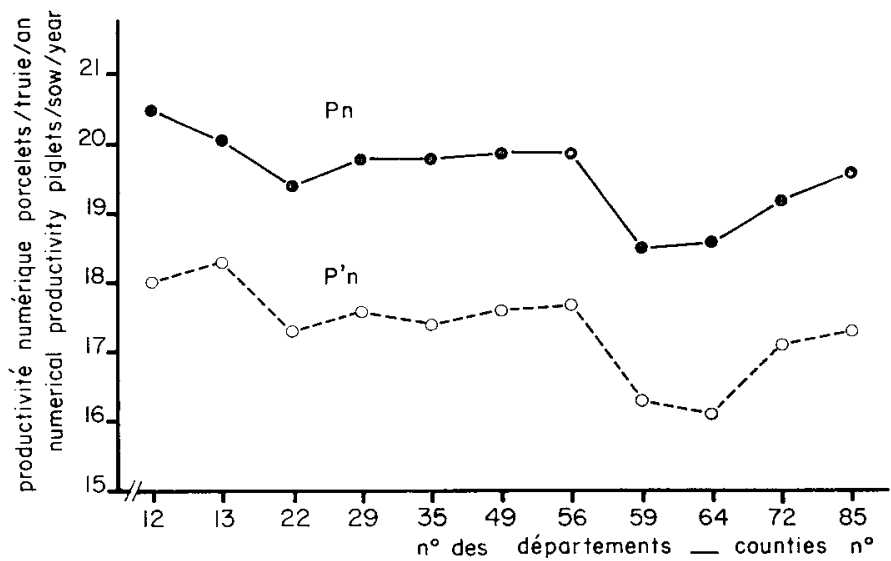

FIG. 7. - Expression de la productivité numérique moyenne annuslle par truie: Pn, par truie productive; $P^{\prime} n$ par truie présente $\left(P^{\prime} n=17,54\right)$ en 1978 .

Expression of the average anmual productivity per sow: $P n$, per productive sow; $P$ 'n per sow present in the herd. ( $P^{\prime} n=17,45$ in 1978$)$.

\section{Discussion}

Les principaux résultats observés permettent de souligner toute l'importance de la mesure des performances des truies par la gestion technique. Par ailleurs, on constate des valeurs très proches entre la moyenne nationale et la valeur calculée sur l'effectif des i I départements de référence.

La réduction de la durée d'allaitement apparaît comme une évolution importante commune à tous les départements. On peut dire sans doute que ce résultat est la conséquence de l'action de tous les techniciens en faveur de l'adoption du sevrage précoce. Ainsi le sevrage à partir de 3 semaines d'âge bien maîtrisé et sans conséquences sur la reproduction de la truie en troupeau expérimental (Dv- 
RENDAHI, et al., I958; AUMAITRE et RETTAGLIATI, I972; SVAJGR et al., I974; COLE, VARLEY et HUGUES, I975), peut être généralisé au niveau des troupeaux de production. Un progrès notable a été fait pour ramener le sevrage aux environs de 20 à 30 jours d'âge. La pratique du sevrage à jour fixe dans la semaine a été largement adoptée à la suite d'une conduite en bande favorisant l'organisation du travail, 1'adoption des animaux en surnombre à la naissance, et permettant la désinfection et le vide sanitaire des bâtiments de maternité et de sevrage (SCHNEIDER et BRONSCH, I973). Sur la base des résultats déjà observés dans les Bouchesdu-Rhône, on peut encore escompter une réduction de la durée de lactation, notamment dans certains départements comme le Nord et surtout le Finistère où les éleveurs maîtrisent très bien la conduite de la reproduction des truies.

Par contre, les progrès sont plus lents pour réduire la durée improductive qui sépare le sevrage d'une nouvelle fécondation. D'autre part, il est inutile (Moody, SpeEr et HAys, I966; F́tienne et DUEe, I976; WALKER et al., r979) d'allonger la durée entre le sevrage et une nouvelle fécondation, car le gain sur le taux d'ovulation est illusoire face à la perte économique engendrée par l'entretien alimentaire de la truie. Les valeurs moyennes ne présentent pas de signification physiologique (AUMAITRE et al., I976) en raison d'une proportion très importante (5 p. Ioo environ) de truies ayant un écart sevrage-saillie fécondante supérieur à 90 jours, à la suite d'une mauvaise détection des chaleurs, de nombreux retours, un mauvais diagnostic de gestation, des avortements ou fausses gestations (VAN DER HEyde et LIEVENS, I976). Dans ce domaine où l'on dispose de nombreuses techniques basées sur l'utilisation du verrat, le sondage ultra-sonore, certains dosages hormonaux (Bosc, MaRTINAT-BOTTE et NICOI,LE, I977), un progrès très important reste à faire pour leur diffusion et une bonne application par l'éleveur. Il convient de souligner que l'infécondité peut également être attribuée au verrat que l'on incrimine rarement au niveau des élevages (UzU, I979). En effet, une variation de Io à 27 jours pour l'intervalle sevrage-fécondation a été signalée indépendamment de l'âge au sevrage entre troupeaux exploités dans le même département (AumaiTre et al., I973b) sans établir clairement les causes de cette différence.

Enfin une réduction concomitante de la durée de lactation et de l'intervalle sevrage-fécondation est observée au cours des 6 années. La liaison positive entre ces deux paramètres semble indépendante de la truie et sans signification physiologique. En effet, c'est seulement pour un sevrage inférieur à Io jours ou supérieur à 40 jours que cette durée s'allonge (AUMAITRE. I978), I1 semble que les éleveurs qui laissent téter les porcelets le plus longtemps sont en même temps ceux qui surveillent le moins bien le retour en chaleur de leurs truies. Enfin les longues durées de lactation proviennent généralement d'élevages de faible taille, qui manquent de bâtiment de sevrage adapté, d'un effectif suffisant de verrats, ou qui bénificient d'une surveillance réduite.

La taille moyenne de la portée à la naissance peut encore être améliorée, puisque selon Molenat (I 960), la moyenne du nombre de porcelets nés vivants était de I0,7 dans le Herd Book de la race Large White en I959 alors qu'elle n'est que de IO,I6 sur l'ensemble de l'échantillon où les animaux de cette race ou issus d'un croisement Large White $\times$ Landrace sont dominants. Toutefois, il ne faut pas ignorer que l'application du sevrage précoce pent être responsable d'une légère diminution de la taille de la portée à la naissance (AUMAITRE, r978; LEGAULT, I978) et nous n'avons pu tenir compte de cet effet dans notre étude.

On peut penser que l'ensemble des données comprend toujours la même proportion d'animaux primipares et que les comparaisons peuvent être effectuées 
entre années et entre départements. Ainsi, la taille de la portée à la naissance est inférieure en moyenne aux performances des animaux inscrits aux livres généalogiques (LEGAUL, FELGINES et OWEN, I977). Mais la taille de la portée au sevrage mesurée cependant à un âge différent entre 1972 et 1978 a pu également augmenter, grâce à une réduction sensible du taux de la mortalité post-natale. L'amélioration des conditions d'habitat et d'ambiance et l'aménagement intérieur des maternités (DE I,A PORTE DES VAUX et AUMAITRE, I967; ROBERTson et al., I966; AumaItre et al., I973a), peuvent aussi largement expliquer ce progrès. On observe ainsi une réduction des pertes par sous-nutrition, hypoglycémie et écrasement, alors que les soins à la naissance et la surveillance des mise-bas ont presque complètement disparu. En 6 ans, on a réduit l'intervalle entre mise-bas de 2 semaines ( $\mathrm{I} 77$ à $\mathrm{I} 63$ jours) et augmenté la productivité annuelle de $2, \mathrm{r}$ porcelets. Ainsi, on peut estimer globalement que $77 \mathrm{p}$. roo de ce progrès (soit $\mathrm{r}, 6$ porcelet) s'expliquent par la réduction de la durée d'allaitement; la diminution de l'intervalle sevrage saillie fécondante et de la mortalité expliquent 0,5 porcelet en plus par an.

La productivité numérique a fortement progressé en 6 ans mais elle est très variable d'un département à l'autre. Elle reste faible dans le département du Nord qui peut réduire la durée de la lactation et augmenter la taille des portées. La productivité plafonne dans le Finistère et les Côtes-du-Nord en raison d'un âge au sevrage tardif et malgré un écart sevrage-fécondation réduit. Le même phénomène s'observe dans les Bouches-du-Rhône avec un sevrage précoce, mais un intervalle sevrage-fécondation trop élevé. Enfin, la productivité maximum est observée dans les élevages de 1'Aveyron où 1'on enregistre de faibles pertes chez les truies les plus prolifiques du troupeau national, et où l'âge au sevrage et l'intervalle sevrage-fécondation sont en cours de réduction. Des progrès dont la signification économique est très importante en matière de productivité par truie présente restent à accomplir. En effet, la plupart des moyennes départementales (5 sur I I) sont en 1978 voisines de $I 7,5$ porcelets par truie et par an. Une réduction de 1'âge à la puberté peut être recherchée (CARREz et al., I977) mais c'est surtout une meilleure politique de réforme qui mérite d'être promulguée. L'écart, anormalement élevé entre le sevrage et la réforme (6o jours en I978), fait baisser considérablement la productivité (DAGORN et AumaITRE, I979). La surveillance de la reproduction est donc un impératif important pour réaliser de nouveaux progrès; et l'on sait par ailleurs, qu'une proportion importante des truies réformées actuellement est encore fécondable (Josse et al., I979).

Ainsi, la gestion technique qui permet d'attirer l'attention de l'éleveur sur les points faibles de son élevage a fortement contribué à une amélioration spectaculaire des performances techniques et économiques des truies du troupeau français. De tels progrès ont été la conséquence de la mise au point de techniques nouvelles d'élevage et de leur diffusion auprès des éleveurs. L'application généralisée du sevrage précoce, alors que les autres conditions comme le niveau d'alimentation où les cotits d'exploitation ont peu évolué au cours des 6 dernières années, y a largement contribué.

Accepté par publication en janvier 1980.

\section{Remerciements}

A F. DU MESnil du Buisson et à C. LEGauitr pour leur aide efficace dans la prépa ration de ce manuscrit. 


\section{Summary}

\section{Numerical productivity of the sow measured by reproduction and weaning records}

Recording of the performance of sows from breeding and commercial herds is made in France since I969. Birth, farrowing, weaning and culling dates are collected individually as well as the litter size at birth and at weaning. The average values for age at weaning, weaningfertilization interval, litter size have been calculated for each herd and country (Fr $=$ Department) and the data recorded between 1972 and 1978 have been analysed. The mean productivity per sow is then expressed by the number of piglets weaned per sow and per year.

The main results have been obtained from 69 ooo litters in 1972 and 513 ooo in 1978 ; I I départments out of 82 carrying out these measurements in 1978 are controlling more than 10 ooo results each corresponding to $64 \mathrm{p}$. Ioo of the data. The mean length of lactation largely decreased between 1972 and 1978 (fig. 2 ); it was reduced by 1 I days within this 6 -year-period, i.e. 4 I. 8 versus 30.9 days, table 1 ; and the weaning-fertilization interval by 3.2 days (20.2 versus I 7 days). Litter size at birth remained unchanged (IO.I 8 piglets born alive par litter), but the viability increased from 84.3 to $86.9 \mathrm{p}$. Ioo of animals born alive on an average, between 1972 and $197^{8}$.

During this period, the numerical productivity per breeding sow was improved by 2.1 animals. $77 \mathrm{p}$. Ioo of this gain was due to the reduction of lactation length; the improvement of the other parameters, weaning-fertilization interval and viability, leading only to a gain of 0.5 piglet. The productivity calculated for each sow present in the herd between the age of 200 days and culling (fig. 7) is of special value in terms of economics. As a national average for all herds, the productivity equals I 7.54 piglets weaned. It varies considerably according to departments, from I6. I to I 8.3 piglets in 1978 because of an abnormal interval (6o days, on an average) between last weaning and culling.

Application of new techniques for recording the productive performance of sows and current use of early weaning at about 24 days may lead to further improvements of sow productivity.

\section{Références bibliographiques}

AUmaitre A., 1978. Les conséquences zootechniques du sevrage précoce. Journées Rech. porcine en France, 10, 25 I-274, I.N.R.A.-I.T.P., ed., Paris.

Aumaitre A., Salmon-I,Egagneur F., igбi. Influence de l'alimentation complémentaire sur la croissance du porcelet avant le sevrage. Ann. Zootech., 10, I27-140.

Aumaitre A., REtTagliati J., 1972. Age au sevrage chez le porcelet : répercussion sur la productivité des truies et influence sur les jeunes animaux. Journées Rech. porcine en France, 4, 273-286, I.N.R.A.-I.T.P., éd., Paris.

Aumaitre A., Bichon F., Bina I., RetTagliati J., r973a. Importance du milieu environnant (température) dans l'élevage des porcelets. Amélioration et contrôle de l'ambiance dans une maternité utilisée sans paille. Journées Rech. porcine en France, 5, 239-247, I.N.R.A.-I.T.P., éd., Paris.

Aumaitre A., Le Pan J., RetTagliati J., Bina L., Rousseau P., i973b. Application du sevrage à I 2 jours dans 1'exploitation d'un troupeau porcin. Résultats préliminaires et répercussions sur le nombre et le poids des porcelets produits avec un sevrage à 5-6 semaines. Journées Rech. porcine en France, 5, 77-9I, I.N.R.A.-I.T.P., éd., Paris.

Aumaitre A., Dagorn J., Legault C., LF Denmat M., I976. Influence of farm management and breed type on sow's conception-weaning interval and productivity in France. Lives. Prod. Sci., 3, 75-83.

Bosc M. J., Martinat-Botte F., Nicolite A., I977. Comparaison de quelques techniques de diagnostic de gestation chez la truie. Journées Rech. porcine en France, 9, 33-37, I.N.R.A.I.T.P., éd., Paris.

TE BRAKE J. H. A., I978. The assessment of the most profitable length of lactation for producing piglets of $20 \mathrm{~kg}$ body weight. Lives. Prod. Sci., 5, 8I-94. 
13tyu. A., I976. La rentabilité des productions porcines. Revue Agric. Bruxelles, 27, 45-94.

Carrez S., Treil F., Duḱ P. H., Acmaitre A., i977. Influences sur la puberté de la truie, de sa période de naissance et de sa durée d'allaitement. Ann. Zootech., 26, 62 I-625.

COLE D. J. A., VARleiy M. A., Hughes P. E., I975. Studies on sow reproduction. The effect of lactation length on the subsequent reproductive performance of the sow. Anim. Prod., $20,40 \mathrm{I}-406$.

D.GORN J., 1975. Le programme national mécanographique de gestion technique des troupaux de truies. Journées Rech. porcine en France, 7, 3-I4, I.N.R.A.-I.T.P., éd., Paris.

DAGORN J., Ferradixi MI, DF JUBEcotrt D., i978. Conduite et gestion d'un atelier porcin. Techniporg., 1, B I-B 41

Dyrendahi. s., Olsson B., BJorek G., Eml.tess $\mathrm{T}$., I958. Artificial raising of baby pigs. Part II. Additional experiments including the effect of early wearning on the fertility of sows. Act. A gri. Scand., 8, I-I9.

Exgrish P., Smith W., MAC LEAx A., I977. The sow-improving her fertility. Farming Press Ltd Ipswich, 3І I pp.

ENGELKE F', I969. Die Kosten der Ferkelerzeugung in Abhängigkeit vom Absatzalter. Schweinzucht $u$ Schweinemast, 17, 281-282.

Étienne M., Duíe P. H., i 976 . Durée de l'intervalle sevrage-cestrus et prolificité des truies : étude statistique de l'influence de divers paramètres de reproduction. Journées Rech. porcine en France, 8, I 33-1 38, I.N.R.A.-I.T.P., éd., Paris.

Josse J., Lf, Denmat M., Martinat-Botte F., Sallikifr J., Vannilik P., Valdelet J. C., 1979. A propos d'une enquête sur les causes de réforme des truies. Journées Rech. porcine en France, 11, 355-363, I.N.R.A.-I.T.P., éd., Paris.

LEGauli C., Felgines C., OWEN J., I977. Analyse statistique des composantes de la productivité numérique des truies de quatre races françaises. Journées Rech. porcine en France, 9 , 57-62, I.N.R.A.-I.T.P., éd., Paris.

Legalit C., Aumaitre A., DU Mesnil, du Buisson F., r975. The improvement of sow productivity, a review of recent experiments in France. Livcs. Prod. Sci, 2, 235-246.

LEGAYLT C., I978. Analyse des composantes de la productivité numérique des truies. Ann. Zootcch., 27, 457-470

Maury Y., Palisse M., Poline F., i 973. Sevrage précoce. Résultats obtenus simultanément selon plusieurs durées de lactation dans un troupeau expérimental. Journées Rech. porcine en France, 5, 263-266, I.N.R.A.-I.T.P., éd., Paris.

DU Mesnil du Buisson F., Mauleon P., Jondet P., i97i. Maîtrise de l'cestrus de la truie. Rec. Méd. l'ét., 146, i $203-1224$.

MOODY N. W., SPEER V. C., HAYS V. W., I966. Reproductive performance of sows bred during the first oestrus following early weaning. J. Anim. Sci., 25, I272 Abs.

Mulenat M., I960. Performances d'élevage des truies inscrites au Herd-book du porc Large White, années 1956 à 1959. Bull. Tech. Inf, Paris, 155, 769-801

ORtavant R., Thibatit C., r97I. Productions animales : Reproduction. Bull. Tech. Inj. Paris, 257, 77-78.

Phouft A., Renoux E., Ferradini M., Dagorn J., i976. I,e prix de revient en production porcine. Facteurs de variation et de progrès. Journées Rech. porcine cn France, 8, E 3, E I 8, I.N.R.A.-I.T.P., éd., Paris.

DE I,A Pokte des VAUX H., Aumaitre A., i 967 . Influence du milieu sur l'évolution de la température rectale et la glycémie chez le porcelet nouveau-né. Ann. Zootech., 16, 235-245.

Robertson J. B., Laird R., Hall J. K. S., Forsyorfi R. J., Thomson J. M., Walker-Love J., I966. A comparaison of two indoor farrowing systems for sows. Anim. Prod., 8, I 7 l-1 78 .

Rozier Abbé, I785. Cours complet d'agriculture. In LAURans R., 1975. L'élevage des porcs à l'époque médiévale. L'Homme et l'Animal. Ethnozootechnie, 1, 523-534.

SALMON-LFGAGNEUR E., JOUANDFT C., I962. Sur le sevrage des porcelets à 1'âge de 5 semaines. Ann. Zootech., 11, 3I I-3I3.

SELF H. L., GRUMmer R. H., 1958. The rate and economy of pig gains and the reproductive behaviour in sows when litters are weaned at 10 days, 2 I days or 56 days of age. J. Anim. Sci., 17, 862-868.

SvajgR A. J., Hays V. W., Cromweir. G. L., DUTT R. H., 1974. Effect of lactation duration on reproductive performance of sows. J. Anim. Sci., 38, I00-105.

Schneider D., BRonsci K., 1973. Einfluss der Stallbelegung nach der sog. Fliessbar:d .... und Rein - Raus - Methode auf die Ferkelaufzucht. Züchtungskunde, 45, 53-6o. 
UzU G., I 979. Influence du verrat sur les principaux paramètres de la productivité du troupeau et sur la durée de gestation. Ann. Zootech, 28, 315-323.

VAN DER HEYDE H., LIFVENS R., I976. Reproduction des truies en fonction de diverses durées de lactation 4. Taille de la portée et nombre de porcelets par truie et par an. Rcvue Agric. Bruxelles, 27, I I 45-I I67.

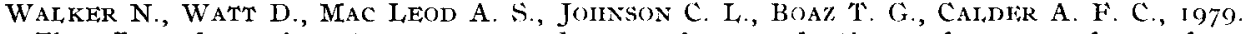
The effect of weaning at 10,25 or 40 days on the reproductive performance of sows from the first to the fifth parity. J. Agri. Sci., Canibridge, 92, 449-456. 